FedUni ResearchOnline

https://researchonline.federation.edu.au

This is the author's accepted version of the following publication:

Allami, R., Stranieri, A., Balasubramanian, V \& Jelineck, H. (2017).

A count data model for heart rate variability forecasting and

premature ventricular contraction detection. Signal Image and Video

Processing, 11(8), 1427-1435.

The version displayed here may differ from the final published version.

The final publication is available at:

https://doi.org/10.1007/s11760-017-1103-x

Copyright (c) 2018, Vietnam Academy of Science and Technology (VAST) and Springer Nature Singapore Pte Ltd. 


\title{
A count data model for heart rate variability forecasting and premature ventricular contraction detection
}

\author{
Ragheed Allami $^{1}$. Andrew Stranieri ${ }^{1}$. Venki Balasubramanian ${ }^{1}$. \\ Herbert F. Jelinek ${ }^{2}$
}

Received: date / Accepted: date

\begin{abstract}
Heart rate variability (HRV) measures including the standard deviation of inter-beat variations (SDNN) require at least five minutes of ECG recordings to accurately measure HRV. In this paper, we predict, using counts data derived from a 3 minute ECG recording, the five minute SDNN and also detect premature ventricular contraction (PVC) beats with a high degree of accuracy. The approach uses counts data combined with a Poisson- generated function, that requires minimal computational resources and is well suited to remote patient monitoring with wearable sensors that have limited power, storage and processing capacity. The ease of use and accuracy of the algorithm provides opportunity for accurate assessment of HRV and reduces the time taken to review patients in real-time. The PVC beat detection is implemented using the same Count Data model together with knowledge-based rules derived from clinical knowledge.
\end{abstract}

Keywords Heart rate variability forecasting . RR interval $\cdot$ SDNN $\cdot$ Premature ventricular contraction

\section{Introduction}

Heart Rate Variability (HRV) is the variation in the inter-beat interval, computed in milliseconds from normal $R$ peaks in an electrocardiograph (ECG) trace, to the next normal $\mathrm{R}$ peak 18 . HRV has been used to predict sudden cardiac death, hypertension and heart failure [16] 32. Also, HRV investigations have associated the risk of cardiovascular disease with chronic stress states 31. According to Nabil, HRV represents the most important feature that can be employed in heart

Ragheed Allami

1 Centre for Informatics and Applied Optimisation, Federation University,Ballarat,3353, Australia

E-mail: ragheed.dawood@yahoo.com arrhythmia detection 26. HRV is typically measured using time-domain, frequency-domain and nonlinear measurements. The root mean square of successive differences (RMSSD) is a common time-domain method however this varies with heart rate 6 . Another time domain feature, the standard deviation of normal to normal intervals(SDNN) varies less with heart rate fluctuations and represents the over-all (both long-term 24 hours and short-term 5 minutes) variation within the $\mathrm{RR}$ interval series [6. SDNN has proven to be important clinically as it allows risk identification of adverse cardiac events 39 13 23. Other time domain measures, include NN50, the number of successive cycles differing more than $50 \mathrm{~ms}$. The standard deviation of the average RR cycles assessed over non-overlapping 5 minutes segments (SDANN) for 24 hours is another time domain measure that is particularly stable [6]. Measures of HRV are affected by heart rate and other local variations so readings of at least five minutes have been recommended 6]. However, the potential clinical benefit inherent in raising alarms early have led to many investigations attempting to find HRV measures in ultra short time frames 34. Numerous investigations that analyse short-term HRV indices using very short time frames of 10-30 sec and, 1 to 3 minute ECG traces have found that the RMSSD is a reliable measure and correlates to RMSSD results over longer ECG traces. However, this is not the case for SDNN or SDANN, which are sensitive to length of recording. There has been no publication outlining a reliable way to model long term RR interval data from short ECG recordings that provide accurate long term SDNN or SDANN results. This study presents an algorithm that uses count data from 3 minute ECG traces to reliably forecast HRV measures for recordings of 5 minute duration. 
Predicting the five-minute HRV with three minutes of RR data is useful in settings where patients safety or distress is paramount as well as shortening consultation times. Screening programmes that use HRV, such as those advocated by [17] can be less stressful to patients and be more cost effective if three minute rather than five minute readings are required. Risk assessments based on HRV in emergency settings such as those advocated by 12 can then also be performed more rapidly for enhanced patient safety. Low HRV extracted from 2 minute traces has been shown to be associated with sudden cardiac death 24 and many other conditions. However a review of HRV studies of short traces (seconds to 3 minutes) revealed large variations from recommended norms 28, which precludes the use of short traces in clinical settings. The motivation underpinning the current study is to discover a mechanism to transform short ECG traces to accurately forecast the 5 minute HRV measures so that the accepted norms derived from the 5 minute HRV can be applied.

In recent years, real-time HRV patient monitoring systems have emerged that use a wireless protocol to stream data from sensors to a nearby, battery operated device (e.g. smartphone) that re-transmits the data to a remotely located server [3]. Algorithms that process the data to raise alarms ideally, execute on devices close to the sensor to avoid delays due to connectivity problems or computational resource constraints [35]. Devices for wearable sensors are predominately portable and hence, battery powered. The execution of complex algorithms and transmission data from these devices consume considerable power and therefore require computationally simple and data efficient algorithms that calculate HRV measures.

The algorithm presented here achieves high accuracy for the RMSSD, SDNN and SDANN forecasts with minimal computational resources because it increments frequency counts of RR intervals as ECG data streams in, and uses these counts to update HRV measures. The frequency counts generated for HRV forecasting can also be used for detecting premature ventricular contraction (PVC), which may be a serious heart condition if detected at frequencies above six per minute.

Although frequency power domain measures of HRV have been found to be powerful, they are computationally expensive 36] and therefore have not been explored in this work.

\section{Related work}

Measures of HRV are affected by respiration frequency, heart rate and other local variations so readings of five minutes have been recommended especially for time and frequency domain measures 6. This is to have a long enough trace for meaningful analysis and avoid non-stationarity effects. The potentially clinical benefit inherent in raising alarms quickly following onset of arrhythmia have led to many investigations attempting to find HRV measures in short and ultra short time frames. For example, Smith et al. 34 identified over 70 HRV measures that had been used for short (30 beats) HRV analysis. Their study demonstrated that shortterm HRV assessment, if able to be calculated quickly and accurately, has the potential to be a good noninvasive diagnostic tool. However, short-term HRV measures require personalised validation before they can be applied to short-term HRV analysis 34 .

Along a similar vein, Esco and Flatt[10] evaluated the agreement of the RMSSD index under ultra-short term conditions with athletes under resting and postexercise conditions and concluded that 60 seconds may be an acceptable recording time for RMSSD but may not accurately represent other 5 minute HRV measures. Thong et al. 37 combined two time domain and one frequency domain HRV measures on 10 seconds traces as a surrogate for the five minute HRV to find that 10 second recordings were not accurate, although RMSSD was promising and the high frequency (HF) power required further investigation.

Taking a longer time frame, Chang et al. 7] compared three minutes to five minutes HRV time and frequency domain measures. The time-domain results indicated that RMSSD and pNN50 analyses were approximately similar at the third and fifth minute but SDNN results were significantly different. In frequency domain measurements, Chang et al. [7] found that low frequency/high frequency power domain measures also varied significantly between the three and five minute recording.

Nussinovitch et al. 29] evaluated the reliability of ultra-short term HRV parameters. The method calculated HRV indices for 10 seconds and 1 minute then compared the results to 5 minute measurements of HRV. Along a similar vein to other work, they concluded that the RMSSD measure seems reliable for analysing HRV from 10 second or 1 minute ECG recordings but this is not the case for SDNN. Frequency domain parameters require longer recording time depending on the frequency range of interest.

None of the studies that investigated the correlation of five-minute HRV using ECG traces shorter than five minutes considered the computational cost of calculating HRV measures. As described above, computational costs must be taken into account to prolong battery life when deploying HRV forecasts in a remote patient monitoring system with wearable sensors and mobile devices. An Android based patient monitoring system that uses wearable heart sensors to identify three types 
of cardiovascular diseases in real-time is presented by 31. They used four time-domain features heart rate, SDNN, RMSSD and pNN50 to detect stress states following five-minute ECG traces but did not report energy consumption or attempt to determine HRV from shorter recordings.

The approach advanced here for determining HRV measures relies on counts of RR intervals. These counts can also be used to detect premature ventricular contraction (PVC), a serious cardiovascular condition that can lead to life-threatening conditions. The instant recognition of life-threatening cardiac arrhythmias is a challenging problem of clinical significance [19]21] 20]. In particular, PVCs have been found to be related to mortality when linked with myocardial infarction[15] and has been shown to be associated with caffeine consumption or stress 1 .

Although, several studies employed RR data to classify and detect PVC beats [26], approaches that are sufficiently accurate and can execute rapidly for fast realtime PVC detection have proven to be elusive. Knowledge based rules have classified and detected heart beats based on a sliding window technique 38] with positive predictive accuracy and sensitivity values of $86.54 \%$ and $87.27 \%$ respectively for PVCs.

A low complexity algorithm for real-time PVC detection that applied a template matching approach for detection of PVC beats from the MIT-BIH Arrhythmia database 19] provided robust results of $98.2 \%$ accuracy and $93.1 \%$ sensitivity. Though accurate, the template matching study requires the detection of the QRS complex and $\mathrm{T}$ waves in real-time for five minutes of training.Similar results were obtained with an Android monitoring system based on wearable heart sensors to classify and detect PVC beats from RR intervals and applying knowledge-based rules 31 .

The detection method presented in the current study uses frequency counts but requires the detection of only the QRS complex without the $\mathrm{T}$ wave, and only for three minutes rather than five, to achieve comparable PVC detection results. Further, the approach advanced here is computationally simpler than template matching and is well suited to execution on power-constrained devices because the additional detection of $\mathrm{T}$ wave leads to greater power consumption on mobile devices.

Recently many cardiovascular wearable sensor devices such as eMotion Faros 33] can detect QRS waves and stream RR data in real-time so can be deployed directly using the approach advanced here.

Other techniques have been applied to classify PVC beats including nonlinear complexity measures, wavelet transform and sophisticated artificial neural networks 19. Support Vector Machine (SVM) have been deployed to discern PVC from Non PVC beats [4. However, these techniques may not always be technically feasible for real-time processing of ECG data from wearable sensors and mobile devices due to high computational resources, power consumption and signal noise 19 .

\section{Material}

Trials of the forecasting algorithm advanced here were applied to the MIT-BIH Arrhythmia database [25] in keeping with other studies. The MIT-BIH Arrhythmia database contains 48 ECG recordings of 30 minutes duration from different patients sampled at $360 \mathrm{~Hz}$. Some ECG traces are normal whereas others are arrhythmic. The PVC detection algorithm was based on two databases; the MIT-BIH Arrhythmia database was used for testing and the St. Petersburg Institute of Cardiological Technics (INCART) database 11 was used for measuring the generalization capability performance of the method. INCART database contains 75 annotated recordings extracted from 32 Holter records. Each record is 30 minutes long and consists of 12 standard leads, each sampled at $257 \mathrm{~Hz}$. The annotations were produced by an automatic algorithm and then corrected manually, containing over 175,000 annotations in all. To match the MIT-BIH-Arrhythmia database the ECG recordings of the INCART database were resampled to $360 \mathrm{~Hz}$ with a tenth-order low-pass finite-impulse response (FIR) filter 22]. In both databases, beats are annotated by expert cardiologists and their details described on 8 .

\section{Method}

\subsection{Forecasting method}

The count data model has emerged as a powerful statistical tool in many fields including machine learning, pattern recognition, data mining, and bioinformatics 2 . Count data is statistical data that represents frequency counts of the number of distinct data occurrences over a specified interval[5]. Table 1 depicts Count RR data in the short-term for PVC and Non PVC rhythms from two readings from the MIT-BIH Arrhythmia database. In record 119 (PVC rhythm), the 18 most frequently occurring $\mathrm{RR}$ intervals to two decimal places are depicted. An $R R=0.88$ seconds occurred the most often, 47 times. The next most frequent interval was $\mathrm{RR}=0.53$ that occurred 33 times. In contrast, Record 112 (Non PVC rhythm), has an RR interval of 0.70 that occurs 127 times and the next most frequent was 0.71 at 93 times. The raw frequency counts from three minutes are filtered through a Poisson distribution in order to arrive at a prediction of the frequency counts that would occur following five minutes. The Poisson distribution is a single parameter discrete probability distribution that 
Table 1 Count RR data for PVC beats (Record No. 119) and Non PVC beat (Record No. 112) heart trace

\begin{tabular}{lllllllll}
\hline RR data & Record No.119 & Annotation & $\begin{array}{l}\text { Count RR } \\
\text { data }\end{array}$ & RR data & Annotation & $\begin{array}{l}\text { Count RR } \\
\text { data }\end{array}$ & RR data & $\begin{array}{l}\text { Record No. } 112 \\
\text { Annotation } \\
\text { Count RR } \\
\text { data }\end{array}$ \\
\hline 0.88 & $\mathrm{~N}$ & 47 & 1.26 & $\mathrm{~N}$ & 7 & 0.70 & $\mathrm{~N}$ & 127 \\
0.53 & $\mathrm{~N}$ & 33 & 0.56 & $\mathrm{~V}$ & 6 & 0.71 & $\mathrm{~N}$ & 93 \\
0.91 & $\mathrm{~N}$ & 32 & 1.27 & $\mathrm{~N}$ & 6 & 0.69 & $\mathrm{~N}$ & 91 \\
0.90 & $\mathrm{~N}$ & 31 & 1.25 & $\mathrm{~N}$ & 6 & 0.68 & $\mathrm{~N}$ & 86 \\
0.89 & $\mathrm{~N}$ & 30 & 0.83 & $\mathrm{~N}$ & 3 & 0.67 & $\mathrm{~N}$ & 36 \\
0.54 & $\mathrm{~N}$ & 26 & 1.35 & $\mathrm{~N}$ & 3 & 0.72 & $\mathrm{~N}$ & 29 \\
0.86 & $\mathrm{~N}$ & 23 & 1.23 & $\mathrm{~N}$ & 3 & 0.73 & $\mathrm{~N}$ & 22 \\
0.87 & $\mathrm{~N}$ & 22 & 1.34 & $\mathrm{~N}$ & 3 & 0.66 & $\mathrm{~N}$ & 9 \\
0.93 & $\mathrm{~N}$ & 22 & 0.84 & $\mathrm{~N}$ & 3 & 0.74 & $\mathrm{~N}$ & \\
\hline
\end{tabular}

V refers to PVC beat as they annotated on the MIT-BIH Arrhythmia database. N refers to Non PVC beats such as N,L,R,a,F,A, etc. as annotated on the MIT-BIH Arrhythmia database

is appropriate for applications that include the counts of events that occur randomly in a given interval of time 42 . Equation 1 describes the Poisson distribution where $\mathrm{X}$ is the number of events in any given interval, and $\lambda$ is the mean number of events per interval, then the probability of observing $\mathrm{x}$ events in a given interval is given by Equation 1:

$P(X=x)=\exp -\lambda \frac{\lambda^{x}}{x !} \quad x=1,2,3, \ldots \infty$

\subsection{Evaluating forecast accuracy}

The forecast error is defined as $E_{j}=X_{j}-\hat{X}_{j}$, where $X_{j}$ denotes the jth observation and $\hat{X}_{j}$ denotes a forecast of $X_{j}$. Accuracy measures that are based on $E_{j}$ are therefore scale-dependent and cannot be used to make comparisons between series that are on different scales. The most commonly used scale-dependent measures are based on the root mean squared error (RMSE) [14].

$R M S E=\sqrt{\frac{\sum_{j=1}^{n}\left(E_{j}\right)^{2}}{n}}$

The traditional equation for the mean $\mathrm{RR}$ is given as:

$\mu=\frac{\sum_{i=1}^{n} X_{i}}{n}$

while the mean count $\mathrm{RR}$ is given as:

$\mu C=\frac{\sum_{i=1}^{n}(\text { RRdatai } * C R R d a t a i)}{n}$

The traditional equation for SDNN is :

$S D N N=\sqrt{\frac{\sum_{i=1}^{n}\left(X_{i}-\mu\right)^{2}}{n-1}}$

whereas the SDNN equation with count RR data is:

$S D N N c=\sqrt{\frac{\sum_{i=1}^{n}((\text { RRdatai } * \text { CRRdatai })-\mu)^{2}}{n-1}}$

\subsection{Proposed RR data forecasting technique}

The forecasting technique is implemented in five steps as described in the algorithm below.

Input: ECG data

Output: Predicted SDNN, RMSNN and Mean RR

Step 1: Apply real-time Pan and Tompkins QRS 30. detection algorithm to extract RR data for three minutes

Step 2: Generate the RR frequency counts on RR to two decimal places(RR data histogram)

Step 3: Apply Poisson probability to the RR counts in the $\mathrm{RR}$ data histogram to estimate the RR counts after another 2 minutes

Step 4: Combine the 3 min counted RR data histogram to the predicted 2 min counted RR data histogram to form the predicted 5 minutes RR data histogram

Step 5: Use equation (4 and 6) to calculate Mean and SDNN

Accordingly, we compared the forecasts generated using the algorithm above with forecasts obtained with an Elman recurrent neural network. Elman network [9] is widely used in time series prediction 41. As outlined above, the RR counts can also be used to detect $\mathrm{PVC}$ in real-time described in the next section.

\subsection{PVC detection technique}

The fact that PVC beats have a QRS pattern wider than Non PVC beats allows identification of these beats relatively easily and counts of RR intervals can be expected to be significantly different [26] 27]. The PVC detection technique was executed in five main steps as demonstrated in the algorithm below. First, the realtime QRS detection algorithm 30] was applied to extract $\mathrm{RR}$ data for three minutes. In the second step, RR counts were generated in the same way as described for the forecasting algorithm. In the remaining steps, knowledge rules were derived from 19 [38 to detect PVC from Non PVC beats. The algorithm for the detection 
of PVC and Non-PVC beats is as follows:

Input: 3 min ECG data

Output: Classification of each beat as PVC or Non PVC

Step 1 and 2: As per forecasting algorithm above

Step 3: Sort counted data according to the count field in descending order

Step 4: Select the first 6 RR data (i.e the most frequent $\mathrm{RR}$ intervals) and calculate the Mean from those

Step 5: For each RR data point, if the RR data $>$ Mean and the Absolute difference value between ( $R R$ data and Mean) $>10 \mathrm{~ms}$ then the RR data is PVC else the $\mathrm{RR}$ data is Non PVC

\subsection{Power consumption}

In this study we used the Samsung Galaxy J1 as a reference to measure energy consumption in Joules. The Samsung Galaxy J1 motherboard consists of a quadcore Cortex-A7 CPU clocked at up to $1.2 \mathrm{GHz}$ with about 5 Watts power, 1GB Ram, OS Android 6.0, and has a Bluetooth module. In addition, Samsung Galaxy J1 has a $1500 \mathrm{mAh}$ rechargeable battery that can work up to 6 hours $(3 \mathrm{G})$. The energy consumption has been estimated for the runtime HRV and PVC methods by analyzing the number of operations implemented by the micro-controller unit; the number of multiplications, summations, divisions and logical comparisons. These operations have been converted to number of cycles.

\section{Result}

\subsection{Forecasting five minute HRV results}

The counts for a single record No. 234 illustrate algorithm outputs. For this patient, 75 beats occurred with an interbeat interval of 0.64 seconds in three minutes and 113 beats occurred with the same interval over five minutes. The algorithm presented here predicted that 111 beats of 0.64 seconds duration would occur in the five minutes. Overall, for that patient, the algorithm finds that the predicted counts from three minutes of data are close to the five minutes counts actually observed. A new interval, 0.69, appeared once in the actual five minutes count that was not present in the three minutes data and therefore not predicted. Interval 0.66 seconds occurred 32 times in the 3 minutes and was predicted to occur 57 times over 5 minutes. However instead, it occurred 67 times. Table 2 illustrates that the SDNN calculated from the 3 minutes is quite close to the SDNN calculated using the Count-Poisson method from the five minutes actual data. A t-test comparing the actual five minute SDNN with the three minutes SDNN $(t=-2.11893$. $p<0.05)$ confirm previous studies that report significant differences. In contrast, there was no significant difference between the predicted and actual five minute SDNN ( $\mathrm{t}-$ value=0.1158. $\mathrm{p}>0.01$ ). Table 3 illustrates that the SDNN is also similar for the same data using the Elman network $(t=0.690, \mathrm{p}>0.01)$. However, the result of overall Root Mean Square Error (RMSE) between actual and predicted SDNN values for the Poisson model was 3, whereas the result of the overall RMSE applying the Elman network was 8 . The results indicated that the Poisson model achieved a higher prediction accuracy $97 \%$ than the Elman network $92 \%$. Therefore, the possible accurate prediction of SDNN from 3 minute recordings compared to the longer recording periods of the MIT-BIH data decreased the computation time from 24 hours to 14.40 hours, which leads to decreased energy consumption for battery-operated devices.

\subsection{PVC detection results}

The performance of the suggested PVC and Non PVC detection algorithm was measured by sensitivity (Se), positive predictive $(+\mathrm{P})$ and accuracy $(\mathrm{Ac})$ for each of 10, non-overlapping, 3 minute segments during 30 minutes. Overall testing and validation results of the proposed algorithm are illustrated in Table 4. The PVC detection results were compared with three published methods where a part or the full MIT-BIH Arrhythmia database were used 31 19 38. Table 5 illustrates the PVC method presented here provided better sensitivity and positive predictive to detect PVCs. Although the algorithm in 19 recognized PVCs beat-by-beat so it may better meet the real-time analysis requirements but the proposed algorithm provided much better sensitivity and positive predictive. Also, the count data model can recognize PVCs in real-time based only on RR data that leads to low computational processing time.

\subsection{Energy consumption results}

Energy consumption was estimated for the runtime HRV and PVC methods by analyzing the number of operations implemented by the micro-controller unit. The energy consumption is comparable with the algorithms listed in Table 6 and is particularly good given that the algorithm executes in real-time and increases battery life using, minimal CPU requirements, less time and power consumption.

Energyconsumption $=$ Power $*$ Runtime 
Table 2 Actual and predicted SDNN for MIT-BIT Arrhythmia database records based on Poisson method

\begin{tabular}{|c|c|c|c|c|c|c|c|c|c|}
\hline $\begin{array}{l}\text { Rec. } \\
\text { No. }\end{array}$ & $\begin{array}{l}\text { Three } \\
\text { min } \\
\text { SDNN } \\
(\mathrm{ms})\end{array}$ & $\begin{array}{l}\text { Actual } \\
\mathrm{SDNN}(\mathrm{ms}) \\
(5 \mathrm{~min})\end{array}$ & $\begin{array}{l}\text { Predicted } \\
\mathrm{SDNN}(\mathrm{ms}) \\
(5 \mathrm{~min})\end{array}$ & RMSE & $\begin{array}{l}\text { Rec. } \\
\text { No. }\end{array}$ & $\begin{array}{l}\text { Three } \\
\text { min } \\
\text { SDNN } \\
(\mathrm{ms})\end{array}$ & $\begin{array}{l}\text { Actual } \\
\mathrm{SDNN}(\mathrm{ms}) \\
(5 \mathrm{~min})\end{array}$ & $\begin{array}{l}\text { Predicted } \\
\mathrm{SDNN}(\mathrm{ms}) \\
(5 \mathrm{~min})\end{array}$ & RMSE \\
\hline 100 & 25.2 & 38.5 & 35.2 & 2.33 & 201 & 144.7 & 178.2 & 66.8 & 0.14 \\
\hline 101 & 48.6 & 55.2 & 48.1 & 5.02 & 202 & 63 & 67 & 155 & 1.63 \\
\hline 102 & 38.5 & 76.8 & 73.4 & 2.40 & 203 & 199 & 157.3 & 64 & 0.14 \\
\hline 103 & 34 & 69 & 76.5 & 5.30 & 205 & 12 & 64.2 & 352.3 & 11.81 \\
\hline 104 & 40.9 & 86 & 80.2 & 4.10 & 207 & 156 & 369 & 147.3 & 1.13 \\
\hline 105 & 72.3 & 105.2 & 97.4 & 5.52 & 208 & 116.1 & 148.9 & 32.7 & 0.85 \\
\hline 106 & 210.9 & 190.3 & 190.9 & 0.42 & 209 & 33.6 & 33.9 & 122.7 & 0.78 \\
\hline 107 & 35.3 & 72.5 & 74.9 & 1.70 & 210 & 109.4 & 123.8 & 42 & 0.35 \\
\hline 108 & 45.3 & 100.2 & 90.9 & 6.58 & 212 & 39.6 & 42.5 & 44.6 & 0.42 \\
\hline 109 & 41.3 & 74.2 & 75.1 & 0.64 & 213 & 19.3 & 44 & 189.5 & 2.62 \\
\hline 111 & 29.5 & 30.8 & 30.3 & 0.35 & 214 & 135.6 & 193.2 & 79.9 & 0.99 \\
\hline 112 & 14.5 & 25.4 & 25 & 0.28 & 215 & 60.5 & 78.5 & 90.3 & 0.64 \\
\hline 113 & 73 & 140.2 & 135.8 & 3.11 & 217 & 56.7 & 89.4 & 129.3 & 0.49 \\
\hline 114 & 93.6 & 84.2 & 80.6 & 2.55 & 219 & 144.6 & 130 & 64 & 4.60 \\
\hline 115 & 62.6 & 123.5 & 120.2 & 2.33 & 220 & 27.3 & 70.5 & 190.3 & 7.85 \\
\hline 116 & 61 & 80 & 80.8 & 0.57 & 221 & 194.5 & 201.4 & 79.2 & 0.78 \\
\hline 117 & 62.6 & 134.8 & 130.9 & 2.76 & 222 & 52.5 & 80.3 & 70.3 & 1.91 \\
\hline 118 & 30.5 & 66.2 & 64.9 & 0.92 & 223 & 75.7 & 73 & 204 & 5.87 \\
\hline 119 & 200.2 & 249.7 & 243.5 & 4.38 & 228 & 124.8 & 212.3 & 97.4 & 4.88 \\
\hline 121 & 43 & 54 & 55.3 & 0.92 & 230 & 52.2 & 90.5 & 271.4 & 12.45 \\
\hline 122 & 50 & 29 & 30.1 & 0.78 & 231 & 207.6 & 289 & 460 & 12.73 \\
\hline 123 & 115.6 & 167.4 & 167.8 & 0.28 & 232 & 205.6 & 478 & 110.1 & 8.98 \\
\hline 124 & 33.4 & 56.3 & 56.9 & 0.42 & 233 & 125.6 & 122.8 & 20 & 3.82 \\
\hline 200 & 166.3 & 123.6 & 125 & 0.99 & 234 & 14.1 & 14.6 & 66.8 & 0.14 \\
\hline
\end{tabular}

Table 3 Actual and predicted SDNN for MIT-BIT Arrhythmia database records based on Elman method

\begin{tabular}{|c|c|c|c|c|c|c|c|c|c|}
\hline $\begin{array}{l}\text { Rec. } \\
\text { No. }\end{array}$ & $\begin{array}{l}\text { Three } \\
\text { min } \\
\text { SDNN } \\
(\mathrm{ms})\end{array}$ & $\begin{array}{l}\text { Actual } \\
\mathrm{SDNN}(\mathrm{ms}) \\
(5 \mathrm{~min})\end{array}$ & $\begin{array}{l}\text { Predicted } \\
\mathrm{SDNN}(\mathrm{ms}) \\
(5 \mathrm{~min})\end{array}$ & RMSE & $\begin{array}{l}\text { Rec. } \\
\text { No. }\end{array}$ & $\begin{array}{l}\text { Three } \\
\text { min } \\
\text { SDNN } \\
(\mathrm{ms})\end{array}$ & $\begin{array}{l}\text { Actual } \\
\mathrm{SDNN}(\mathrm{ms}) \\
(5 \mathrm{~min})\end{array}$ & $\begin{array}{l}\text { Predicted } \\
\mathrm{SDNN}(\mathrm{ms}) \\
(5 \mathrm{~min})\end{array}$ & RMSE \\
\hline 100 & 25.2 & 38.5 & 30.3 & 5.80 & 201 & 144.7 & 178.2 & 59.9 & 5.02 \\
\hline 101 & 48.6 & 55.2 & 40 & 10.75 & 202 & 63 & 67 & 145.7 & 8.20 \\
\hline 102 & 38.5 & 76.8 & 65.6 & 7.92 & 203 & 199 & 157.3 & 64.8 & 0.42 \\
\hline 103 & 34 & 69 & 65.2 & 2.69 & 205 & 12 & 64.2 & 362.1 & 4.88 \\
\hline 104 & 40.9 & 86 & 77.7 & 5.87 & 207 & 156 & 369 & 138.5 & 7.35 \\
\hline 105 & 72.3 & 105.2 & 85.5 & 13.93 & 208 & 116.1 & 148.9 & 33.5 & 0.28 \\
\hline 106 & 210.9 & 190.3 & 180.7 & 6.79 & 209 & 33.6 & 33.9 & 119 & 3.39 \\
\hline 107 & 35.3 & 72.5 & 71 & 1.06 & 210 & 109.4 & 123.8 & 46.2 & 2.62 \\
\hline 108 & 45.3 & 100.2 & 101.6 & 0.99 & 212 & 739.6 & 42.5 & 50.5 & 4.60 \\
\hline 109 & 41.3 & 74.2 & 65.3 & 6.29 & 213 & 19.3 & 44 & 185 & 5.80 \\
\hline 111 & 29.5 & 30.8 & 20.9 & 7.00 & 214 & 135.6 & 193.2 & 81 & 1.77 \\
\hline 112 & 14.5 & 25.4 & 39.3 & 9.83 & 215 & 60.5 & 78.5 & 90.8 & 0.99 \\
\hline 113 & 73 & 140.2 & 120.6 & 13.86 & 217 & 56.7 & 89.4 & 121.2 & 6.22 \\
\hline 114 & 93.6 & 84.2 & 67.6 & 11.74 & 219 & 144.6 & 130 & 60.3 & 7.21 \\
\hline 115 & 62.6 & 123.5 & 109 & 10.25 & 220 & 27.3 & 70.5 & 194.8 & 4.67 \\
\hline 116 & 61 & 80 & 75 & 3.54 & 221 & 194.5 & 201.4 & 101.2 & 14.78 \\
\hline 117 & 62.6 & 134.8 & 80.8 & 38.18 & 222 & 52.5 & 80.3 & 73 & 0.00 \\
\hline 118 & 30.5 & 66.2 & 60.2 & 4.24 & 223 & 75.7 & 73 & 194.6 & 12.52 \\
\hline 119 & 200.2 & 249.7 & 182.8 & 47.31 & 228 & 124.8 & 212.3 & 101.3 & 7.64 \\
\hline 121 & 43 & 54 & 50.8 & 2.26 & 230 & 52.2 & 90.5 & 280.8 & 5.80 \\
\hline 122 & 50 & 29 & 27.9 & 0.78 & 231 & 207.6 & 289 & 479 & 0.71 \\
\hline 123 & 115.6 & 167.4 & 102 & 46.24 & 232 & 205.6 & 478 & 98.8 & 16.97 \\
\hline 124 & 33.4 & 56.3 & 57.4 & 0.78 & 233 & 125.6 & 122.8 & 12.4 & 1.56 \\
\hline 200 & 166.3 & 123.6 & 128.9 & 3.75 & 234 & 14.1 & 14.6 & 59.9 & 5.02 \\
\hline
\end{tabular}




\section{Discussion}

Remote healthcare monitoring based on mobile devices, requires algorithms that consume less computation resources and power consumption. A forecasting algorithm that can accurately predict the five minutes SDNN parameter from three minutes of data in a computationally simple way has been advanced. The algorithm executes so quickly that it can be revised in real-time as each new data point is sensed. Forecasting HRV can contribute to the early detection of stress states and raising alarms for patients with heart disease or neurological conditions such as Parkinsons disease and elderly subjects 31. Also, it reduces the long-term HRV analysis from 24 hours to 14.40 hours that leads to power savings for both wearable and mobile devices. In comparison with previous published real-time techniques our method is computationally simple and can execute in real-time with high accuracy, sensitivity, and positive predictive compared with other methods. The method in 19] requires the detection of QRS and T waves in realtime. This consumes more processing time and power than the approach advanced here that requires only the QRS and uses counts of RR data. In addition, $T$ wave identification is more complex and therefore leads to greater error. This is especially important for mobile devices. In addition, as illustrated above sensors are emerging that stream RR data. With our algorithm, the $\mathrm{RR}$ data can be processed directly further reducing processing time and resources.

\section{Conclusion}

Previous studies found SDNN results differ significantly between 3 and 5 minutes. The current research advances equations that can predict the 5 minute SDNN from 3 minutes of RR data in real-time using a counts data model. Results indicated that predicted SDNN results were similar to actual SDNN results with an overall accuracy of $97 \%$. This contributes to obtaining optimal short time recording ( 5 minutes) for analysing HRV in short term recordings of less than 5 minutes. Also, this paper illustrates that the counts of $\mathrm{RR}$ intervals used for the HRV forecasts can be used to improve real-time PVC beat detection. Results indicated positive predictive accuracy and sensitivity improvements over existing methods. Furthermore, many investigations proposed that SDNN/RMSSD can be used as surrogate for $\mathrm{LF} / \mathrm{HF}$, making this a convenient way to determine sympathovagal balance 40]. Further research is required to apply the proposed methods within a mobile device to determine clinical utility and computational savings.

Acknowledgements Ragheed Allami expresses his special thanks to the Ministry of Higher Education \& Scientific Research and The University of Technology in Iraq for supporting this work.
Table 4 Overall performance in the testing databases of MIT-BIH Arrhythmia and INCART

\begin{tabular}{llll}
\hline Database & $+\mathrm{P} \%$ & Se $\%$ & Ac $\%$ \\
\hline MIT-BIH & 96.6 & 93.7 & 95.4 \\
INCART & 92.7 & 87.5 & 94.2 \\
\hline
\end{tabular}

Table 5 Comparison between the suggested method and published real-time methods

\begin{tabular}{llll}
\hline Study & $+\mathrm{P} \%$ & Se $\%$ & Ac $\%$ \\
\hline Tsipouras et al. [38] & 86.5 & 87.2 & 94.9 \\
Li et al. [19] & 81.4 & 93.1 & 98.2 \\
Pierleoni et al. [31] & 86 & 87 & 94 \\
Proposed method & 96.6 & 93.7 & 95.4 \\
\hline
\end{tabular}

Table 6 Comparison between the suggested methods and frequency domain method for energy consumption

\begin{tabular}{llll}
\hline Method & $\begin{array}{l}\text { Runtime } \\
\text { (Sec) }\end{array}$ & $\begin{array}{l}\text { Energy } \\
\text { consump- } \\
\text { tion(Joules) }\end{array}$ & $\begin{array}{l}\text { Time } \\
\text { (min) }\end{array}$ \\
\hline QRS detection & 0.032 & 0.016 & - \\
T wave detection & 0.016 & 0.08 & - \\
Frequency domain & 0.060 & 0.30 & 5 \\
Our Forecasting & 0.040 & 0.20 & 3 \\
Our PVC detection & 0.050 & 0.25 & 3 \\
\hline
\end{tabular}

\section{References}

1. Agrafioti, F., Hatzinakos, D.: Ecg biometric analysis in cardiac irregularity conditions. Signal, Image and Video Processing 3(4), 329 (2009)

2. Bakhtiari, A.S., Bouguila, N.: A variational bayes model for count data learning and classification. Engineering Applications of Artificial Intelligence 35, 176-186 (2014)

3. Balasubramanian, V., Stranieri, A.: Performance evaluation of the dependable properties of a body area wireless sensor network. In: Optimization, Reliabilty, and Information Technology (ICROIT), 2014 International Conference on, pp. 229-234. IEEE (2014)

4. Bazi, Y., Hichri, H., Alajlan, N., Ammour, N.: Premature ventricular contraction arrhythmia detection and classification with gaussian process and s transform. In: Computational Intelligence, Communication Systems and Networks (CICSyN), 2013 Fifth International Conference on, pp. 36-41. IEEE (2013)

5. Cameron, A.C., Trivedi, P.K.: Regression analysis of count data, vol. 53. Cambridge university press (2013)

6. task Force of the European Society of Cardiology: Heart rate variability standards of measurement, physiological interpretation, and clinical use. Eur heart J 17, 354-381 (1996)

7. Chang, G.H.L.Y.H., Lin, K.P.: Comparison of heart rate variability measured by ecg in different signal lengths. Journal of Medical and Biological Engineering 25(2), 6771 (2005)

8. physioBank databases: http://www.physionet.org/cgibin/atm/ATM. Last visited on 20 January (2016)

9. Elman, J.L.: Finding structure in time. Cognitive science 14(2), 179-211 (1990) 
10. Esco, M.R., Flatt, A.A.: Ultra-short-term heart rate variability indexes at rest and post-exercise in athletes: evaluating the agreement with accepted recommendations. Journal of sports science \& medicine 13(3), 535 (2014)

11. Goldberger, A.L., Amaral, L.A., Glass, L., Hausdorff, J.M., Ivanov, P.C., Mark, R.G., Mietus, J.E., Moody, G.B., Peng, C.K., Stanley, H.E.: Physiobank, physiotoolkit, and physionet. Circulation 101(23), e215-e220 (2000)

12. Heldeweg, M.L.A., Liu, N., Koh, Z.X., Fook-Chong, S., Lye, W.K., Harms, M., Ong, M.E.H.: A novel cardiovascular risk stratification model incorporating ecg and heart rate variability for patients presenting to the emergency department with chest pain. Critical Care 20(1), 179 (2016)

13. Huikuri, H.V., Stein, P.K.: Heart rate variability in risk stratification of cardiac patients. Progress in cardiovascular diseases 56(2), 153-159 (2013)

14. Hyndman, R.J., Athanasopoulos, G.: Forecasting: principles and practice. OTexts (2014)

15. Iwasa, A., Hwa, M., Hassankhani, A., Liu, T., Narayan, S.M.: Abnormal heart rate turbulence predicts the initiation of ventricular arrhythmias. Pacing and clinical electrophysiology 28(11), 1189-1197 (2005)

16. Jarrin, D.C., McGrath, J.J., Giovanniello, S., Poirier, P., Lambert, M.: Measurement fidelity of heart rate variability signal processing: The devil is in the details. International Journal of Psychophysiology 86(1), 88-97 (2012)

17. Kotecha, D., New, G., Flather, M., Eccleston, D., Pepper, J., Krum, H.: Five-minute heart rate variability can predict obstructive angiographic coronary disease. Heart 98(5), 395-401 (2012)

18. Kranjec, J., Beguš, S., Geršak, G., Drnovšek, J.: Noncontact heart rate and heart rate variability measurements: A review. Biomedical Signal Processing and Control 13, 102-112 (2014)

19. Li, P., Liu, C., Wang, X., Zheng, D., Li, Y., Liu, C.: A low-complexity data-adaptive approach for premature ventricular contraction recognition. Signal, Image and Video Processing 8(1), 111-120 (2014)

20. Liu, C., Liu, C., Shao, P., Li, L., Sun, X., Wang, X., Liu, F.: Comparison of different threshold values $r$ for approximate entropy: application to investigate the heart rate variability between heart failure and healthy control groups. Physiological Measurement 32(2), 167 (2010)

21. Liu, C.Y., Li, L.P., Zhao, L., Zheng, D.C., Li, P., Liu, C.C.: A combination method of improved impulse rejection filter and template matching for identification of anomalous intervals in $\mathrm{rr}$ sequences. $\mathrm{J}$ Med Biol Eng 32(4), 245-249 (2012)

22. Llamedo, M., Martínez, J.P.: Heartbeat classification using feature selection driven by database generalization criteria. IEEE Transactions on Biomedical Engineering 58(3), 616-625 (2011)

23. Loguidice, M.J., Schutt, R.C., Horton, J.W., Minei, J.P., Keeley, E.C.: Heart rate variability as a predictor of death in burn patients. Journal of Burn Care \& Research 37(3), e227-e233 (2016)

24. Maheshwari, A., Norby, F.L., Soliman, E.Z., Adabag, S., Whitsel, E.A., Alonso, A., Chen, L.Y.: Low heart rate variability in a 2-minute electrocardiogram recording is associated with an increased risk of sudden cardiac death in the general population: The atherosclerosis risk in communities study. PloS one 11(8), e0161,648 (2016)

25. Mark, R., Moody, G.: Mit-bih arrhythmia database 1997. URL http://ecgmit. edu/dbinfo. html (1997)
26. Nabil, D., Reguig, F.B.: Ectopic beats detection and correction methods: A review. Biomedical Signal Processing and Control 18, 228-244 (2015)

27. Nguyen, T.B., Lou, W., Caelli, T., Venkatesh, S., Phung, D.: Individualized arrhythmia detection with ecg signals from wearable devices. In: Data Science and Advanced Analytics (DSAA), 2014 International Conference on, pp. 570-576. IEEE (2014)

28. Nunan, D., Sandercock, G.R., Brodie, D.A.: A quantitative systematic review of normal values for short-term heart rate variability in healthy adults. Pacing and Clinical Electrophysiology 33(11), 1407-1417 (2010)

29. Nussinovitch, U., Elishkevitz, K.P., Katz, K., Nussinovitch, M., Segev, S., Volovitz, B., Nussinovitch, N.: Reliability of ultra-short ecg indices for heart rate variability. Annals of Noninvasive Electrocardiology 16(2), 117-122 (2011)

30. Pan, J., Tompkins, W.J.: A real-time qrs detection algorithm. IEEE transactions on biomedical engineering (3), 230-236 (1985)

31. Pierleoni, P., Pernini, L., Belli, A., Palma, L.: An android-based heart monitoring system for the elderly and for patients with heart disease. International journal of telemedicine and applications 2014, 10 (2014)

32. Rodríguez-Liñares, L., Méndez, A.J., Lado, M.J., Olivieri, D.N., Vila, X.A., Gómez-Conde, I.: An open source tool for heart rate variability spectral analysis. Computer methods and programs in biomedicine 103(1), 39-50 (2011)

33. wearable sensor: http://ecg.biomation.com/faros.htm Last visited on 14 February (2016)

34. Smith, A.L., Owen, H., Reynolds, K.J.: Heart rate variability indices for very short-term (30 beat) analysis. part 2 : validation. Journal of clinical monitoring and computing 27(5), 577-585 (2013)

35. Szczepański, A., Saeed, K.: A mobile device system for early warning of ecg anomalies. Sensors 14(6), 11,03111,044 (2014)

36. Tarvainen, M.P., Niskanen, J.P., Lipponen, J.A., RantaAho, P.O., Karjalainen, P.A.: Kubios hrv-heart rate variability analysis software. Computer methods and programs in biomedicine 113(1), 210-220 (2014)

37. Thong, T., Li, K., McNames, J., Aboy, M., Goldstein, B.: Accuracy of ultra-short heart rate variability measures. In: Engineering in Medicine and Biology Society, 2003. Proceedings of the 25th Annual International Conference of the IEEE, vol. 3, pp. 2424-2427. IEEE (2003)

38. Tsipouras, M.G., Fotiadis, D.I., Sideris, D.: An arrhythmia classification system based on the rr-interval signal. Artificial intelligence in medicine 33(3), 237-250 (2005)

39. Vaage-Nilsen, M., Rasmussen, V., Jensen, G., Simonsen, L., Mortensen, L.S.: Recovery of autonomic nervous activity after myocardial infarction demonstrated by shortterm measurements of sdnn. Scandinavian Cardiovascular Journal 35(3), 186-191 (2001)

40. Wang, H.M., Huang, S.C.: Sdnn/rmssd as a surrogate for lf/hf: a revised investigation. Modelling and Simulation in Engineering 2012, 16 (2012)

41. Wang, J., Wang, J.: Forecasting energy market indices with recurrent neural networks: Case study of crude oil price fluctuations. Energy 102, 365-374 (2016)

42. Winkelmann, R.: Econometric analysis of count data. Springer Science \& Business Media (2013) 